\title{
Graves' ophthalmopathy: V. Aetiology of upper eyelid retraction in Graves' ophthalmopathy
}

\author{
Steven E Feldon, Lori Levin
}

\begin{abstract}
The volume of the levator superior rectus muscle complex was determined from computed tomography scans of 152 orbits from 76 patients with Graves' ophthalmopathy. Clinically observed lid retraction and lid lag did not correlate with the levator/superior rectus muscle volume and did not correlate with limitation of vertical eye movements or inferior rectus muscle volume. These results do not support previously published hypotheses that muscle hypertrophy or levator/superior rectus muscle overaction secondary to inferior rectus muscle restriction are major causes of lid retraction and lid lag. Local adhesion of the levator muscle to fixed orbital tissues is postulated to be the most likely basis for the characteristic lid retraction and lid lag of Graves' ophthalmopathy.
\end{abstract}

Retraction of the upper lid is a highly characteristic sign of Graves' ophthalmopathy. The retraction present in primary gaze is called Dalrymple's sign, and that present in down gaze (that is, lid lag) is called von Graefe's sign. Although most signs of Graves' ophthalmopathy can be attributed to inflammatory swelling and fibrosis of the extraocular muscles, ${ }^{1}$ the pathophysiological basis for the lid retraction is not clear. Several possible causes have been suggested. Moses $^{2}$ supports the hypothesis advanced by Eden and Trotter ${ }^{3}$ that overactivity of the sympathetic nervous system due to high levels of circulating thyroid hormone or another unidentified factor stimulates the tonus of the levator muscle, Müller's muscle, or both. Against this hypothesis of chemically induced muscle overaction is a lack of pupillary dilatation or other sign of sympathetic nervous system overactivity. Moreover, lid retraction often persists without hyperthyroidism. Levator overaction may also result from synkinetic activity related to the use of superior rectus muscles in overcoming inferior rectus restriction. ${ }^{4} \mathrm{Grove}^{5}$ has reported on fatty infiltration between levator muscle and Müller's muscle, connective tissue proliferation forming adhesions to levator muscle, and degenerative changes within the levator muscle. He suggested that inflammatory changes with resultant enlargement and fibrosis of the levator muscle may cause the retraction.

Recently Small ${ }^{6}$ reported that hypertrophy of individual muscle fibres contributed to the levator enlargement of Graves' ophthalmopathy. He proposed that this muscle hypertrophy caused 'hyperfunction' of the lid, leading to lid retraction. If Small's hypothesis is correct, then there should be a good correlation between quantitative measurements of superior rectus/ levator muscle volume and clinical measures of lid retraction and lid lag. In the study described here this relationship between levator size and function is evaluated to determine possible pathophysiological mechanisms involved in lid retraction associated with Graves' ophthalmopathy.

\section{Patients and methods}

Computed tomographic scans of the orbits ( 0 to $-15^{\circ}$ angulation relative to the canthal meatal line) were obtained from 152 orbits of 76 patients with Graves' ophthalmopathy. The average age of the patients was $50 \cdot 1$ years (range 12 to 82 ); 14 were males and 62 females. All the patients were clinically euthyroid at the time of their evaluation. Other data from this patient group have been previously reported. ${ }^{7}$

A General Electric 9800 or General Electric 8800 computed tomographic scanner was available for performing the orbital scans. Nonoverlapping, adjacent slices through the entire orbit (including roof and floor) were obtained in the axial plane at $1.5 \mathrm{~mm}$ intervals, except in nine patients in whom other intervals of $5 \mathrm{~mm}$ or less were used. The scans were imaged at window widths of 250 or 500 and at computed tomography numbers of 0 to +20 Hounsfield units. These were quantitatively analysed by an image analyser. ${ }^{8}$ An attached pen enabled contours to be traced and digitised for analysis. Software provided with the image analyser was used to determine relative and absolute areas and volumes of structures by evaluating serial twodimensional sections.

The volume of the superior muscles group included both levator and superior rectus muscle, as they could not be differentiated in the axial scan.

Lid retraction was measured as the amount of exposed sclera superior to the corneal limbus with the patient looking straight ahead. Lid lag was measured in a manner similar to lid retraction, but with the eyes directed downward. Up to 1 or $2 \mathrm{~mm}$ of possible lid retraction or lid lag were ignored in this operational definition in order to provide a constant landmark for quantitative measurement.

\section{Results}

Levator/superior rectus muscle volume was plotted against lid retraction and lid lag (Figs 1 and 2). A correlation performed between superior rectus/levator muscle volume and lid retraction resulted in coefficients of 0.02 with percentage total orbital volume, and 0.12 with absolute volume (see Table I). With lid lag as the clinical variable, correlation coefficients were 


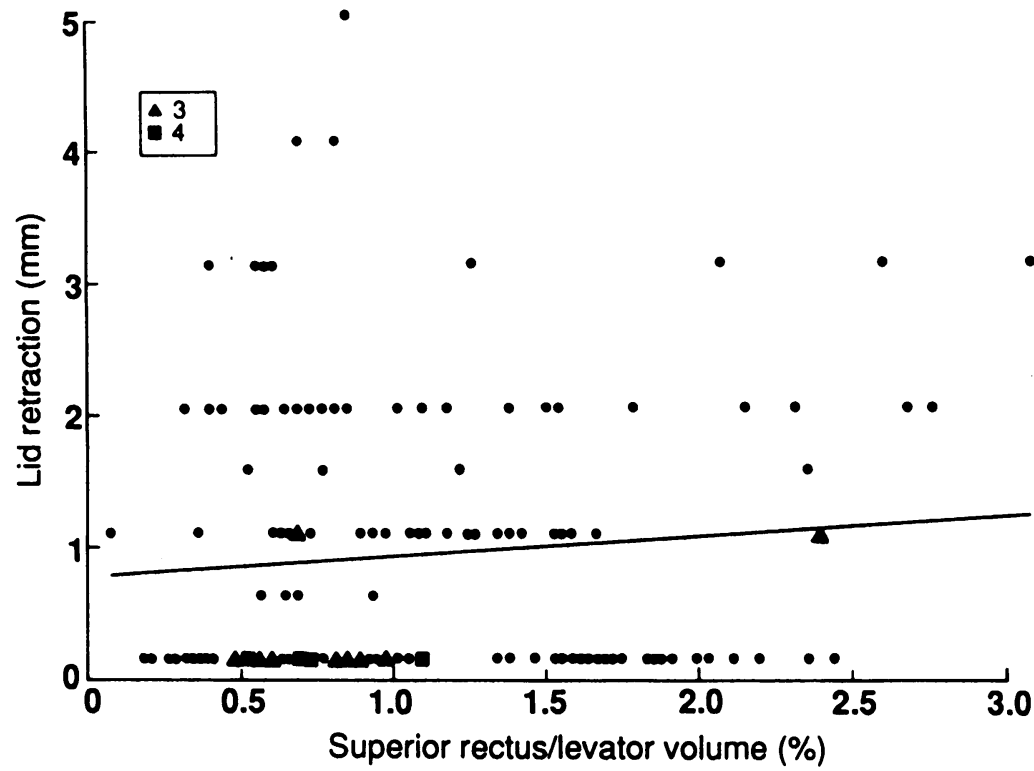

Figure 1 Superior rectus/levator muscle volume is expressed as a percentage of total orbital volume and plotted against lid retraction measured above the superior limbus. Diffuse scatter is demonstrated with almost no correlation. The solid line indicates least squares regression. Legend symbols indicate multiple overlapping points.

0.04 and 0.07 . Similarly, low correlation coefficients $(0.04$ and 0.08$)$ were obtained when lid retraction was tested against relative and absolute volume of the inferior rectus muscle. Correlations of inferior rectus muscle volume with lid lag resulted in coefficients of 0.09 and 0.01 . Correlation coefficients between lid retraction or lid lag and limitation of vertical motility were 0.08 and 0.04 , respectively. None of these values was statistically significant. By comparison, medial rectus volume did correlate with horizontal limitation of motility for this patient group with a coefficient of 0.54 , and vertical limitations correlated with vertical muscle volumes with correlation coefficients between 0.33 and 0.38 (not shown in Table I).

\section{Discussion}

A significant correlation of extraocular muscle volume with extraocular muscle limitation has been shown in prior studies. ${ }^{78}$ At first glance it

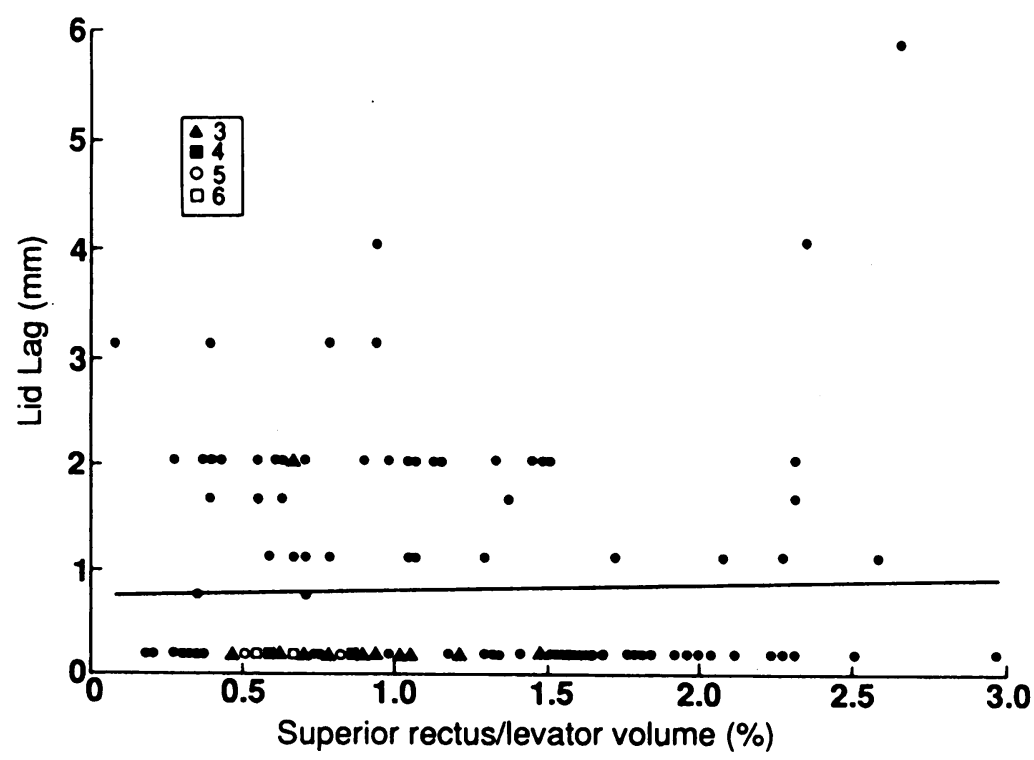

Figure 2 Superior rectus/levator volume (\%) is plotted against lid lag (mm). See Figure 1 legend for further explanation.
Table 1 Correlation of lid findings with extraocular muscle volume and function

\begin{tabular}{|c|c|c|c|c|c|}
\hline & \multicolumn{2}{|c|}{$\begin{array}{l}\text { Superior rectus/ } \\
\text { levator volume }\end{array}$} & \multicolumn{2}{|c|}{$\begin{array}{l}\text { Inferior rectus/ } \\
\text { volume }\end{array}$} & \multirow{2}{*}{$\begin{array}{l}\text { Vertical } \\
\text { limitation } \\
\text { motility }\end{array}$} \\
\hline & $\%$ & $\mathrm{~cm}^{\prime}$ & $\%$ & $\mathrm{~cm}^{i}$ & \\
\hline $\begin{array}{l}\text { Lid retraction } \\
\text { Lid lag }(\mathrm{mm})\end{array}$ & $\begin{array}{l}0.02 \\
0.04\end{array}$ & $\begin{array}{l}0.12 \\
0.07\end{array}$ & $\begin{array}{l}0.04 \\
0.09\end{array}$ & $\begin{array}{l}0.08 \\
0.01\end{array}$ & $\begin{array}{l}0.08 \\
0.04\end{array}$ \\
\hline
\end{tabular}

seems somewhat surprising that lid retraction and lag do not correlate with enlargement of the involved muscle group. However, considering that the superior rectus/levator muscle group is usually substantially increased in volume only in advanced disease, ${ }^{8}$ and that lid retraction/lag are often early signs, perhaps the absence of correlation should have been expected. These findings do not confirm the contention of Small ${ }^{6}$ and of Frueh et $a l^{9}$ that muscle hypertrophy and hyperfunction are related. The possibility that degenerative changes ${ }^{5}$ or disinsertions of the levator ${ }^{9}$ may counteract this relationship cannot be excluded.

Putterman and Urist ${ }^{+}$suggest that a synkinesis of the superior rectus and levator muscles to overcome inferior rectus restriction may frequently cause lid retraction. The lack of significant correlations between lid retraction and either clinical limitation of vertical motility or inferior rectus volume argues against their hypothesis.

Chemically mediated sympathetic nervous system overactivity with Müller muscle overaction may play a part in the development of lid retraction and lid lag, but in this study such sympathetic overactivity is not a consideration because all patients tested were clinically euthyroid.

On the basis of this study the explanation by Grove $^{5}$ indicating that lid retraction and lag may be related to adherence of the levator muscle to adjacent fixed orbital connective tissue and fat seems the most plausible. Such inflammatory changes may be independent of the levator muscle volume and are unrelated either to current thyroid status or to inferior rectus restriction. Of course in individual instances any or all of these possible causes of the observed lid changes may operate.

This work was supported in part by grant EY0 3908 from the National Institutes of Health, by an award from Research to Prevent Blindness Inc, New York, and by core grant EY0 3040 (Biometry Module)

1 Kroll AJ, Kuwabara T. Dysthyroid ocular myopathy: anatomy, histology, and electron microscopy. Arch Ophthalmol 1966; 76: $244-57$

2 Moses RA. The eyelids. In: Moses RA, Hart WM Jr, eds Adler's physiology of the eye: clinical application. St Louis: Mosby, 1987: 1-14.

3 Eden KC, Trotter WR. Lid-retraction in toxic diffuse goitre. Lancet 1942; ii: $385-7$.

4 Putterman AM, Urist $M$. Surgical treatment of upper eyelid retraction. Arch Ophthalmol 1972; 87: 401-5.

5 Grove AS Jr. Upper eyelid retraction and Graves' disease. Ophthalmology 1981; 88: 499-506.

6 Small RG. Enlargement of levator palpebrae superioris muscle fibers in Graves' ophthalmopathy. Ophthalmology 1989; 96: 424-30.

7 Hallin ES, Feldon SE. Graves' ophthalmopathy: II. Correlation of clinical signs with measures derived from computed tomography. Br J Ophthalmol 1988; 72: 678-82.

8 Feldon SE, Weiner JM. Clinical significance of extraocular muscle volumes in Graves' ophthalmopathy: a quantitative computed tomography study. Arch Ophthalmol 1982; 100: 1266-9.

9 Frueh BR, Musch DC, Garber FW. Lid retraction and levator aponeurosis defects in Graves' eye disease. Ophthalmic Surg 1986; 17: 216-20. 